\title{
MATHEMATICAL MODELING OF ELECTROLYTE FILTRATION THROUGH THE POROUS CATHODE BLOCKS DURING ALUMINUM ELECTROLYSIS WITH REGARD INTERBLOCK SEAMS
}

\author{
Anton S. Orlov ${ }^{1}$, Andrey V. Minakov ${ }^{1,2}$, Alexander V. Proshkin ${ }^{1}$ \\ ${ }^{1}$ Siberian Federal University, Kirensky st. 26, Krasnoyarsk, Russian Federation \\ ${ }^{2}$ Institute of Thermophysics SB RAS, Lavrentiev av. 1, Novosibirsk, Russian Federation
}

\begin{abstract}
This article investigates electrolyte filtration in the bottom of the aluminum electrolyzer cathode device using the mathematical modeling. Penetration of molten electrolyte in the heat insulation part of the lining is one of the main reasons of electrolyzer premature shutdown, because it leads to bottom destruction and excessive heat loss. This problem is considered a two-phase filtration of incompressible immiscible liquids in an inhomogeneous non-deformable porous body. The verification of the model on the problem of water filtration pin a porous medium has confirmed its adequacy. With the help of the developed mathematical model the dynamics of the impregnation of the lining of the cathode and electrolyte device defined thermal balance baths. Research has identified the speed of penetration of the melt in the bottom of the bath during service of the electrolyzer.
\end{abstract}

\section{INTRODUCTION}

Although the principles of Hall-Heroult process not changed since their introduction more than 120 years ago, modern requirements for cathodic bottom materials became much more serious than it was back then [1-4]. Designing new electrolyzers raises question of expediency of using various lining materials. It's important to know beforehand, how materials behave themselves, when exposed to corrosive components, during service cathode in electrolyzers [5-6].

Purpose of this article is to develop a mathematical model of the penetration process of electrolyte in cathode of aluminum production electrolyzers. Mathematic modeling is a valuable tool, which can help optimize the existing structure of cathode device lining and save time and effort in the development of new electrolyzers. Modelling is particularly valuable for the thermoelectric analysis of bath, and also allows quickly recalculate new the frozen electrolyte profile and thermal balance, if elements of bottom or their properties are changed.

\section{Statement of the Problem}

During operation of the electrolyzer is continuous filtration of the electrolyte in the bottom of the cathode. The hydrostatic pressure of the electrolyte displaces the air from the pores of lining materials (figure 1). The molten electrolyte is at a temperature of $956{ }^{\circ} \mathrm{C}$ in bath. At $850{ }^{\circ} \mathrm{C}$ (liquidus temperature) electrolyte solidifies and his filtration stops. The parameters for solution of the problem are given in table 1.

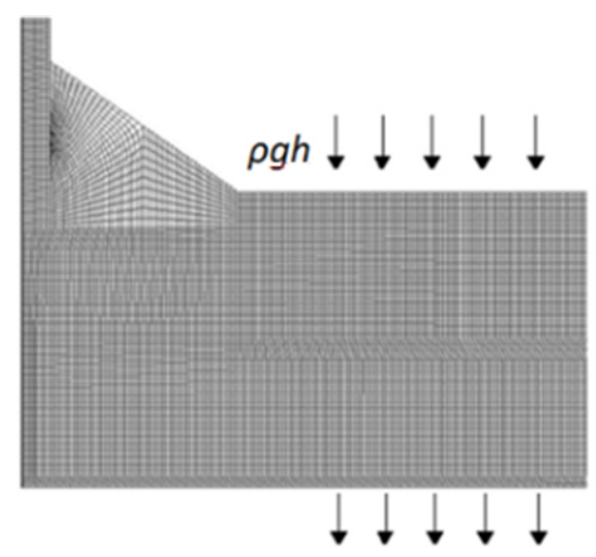

Figure 1. The computational domain and grid.

\footnotetext{
${ }^{a}$ Corresponding author: fogelo@yandex.ru
} 
Table 1. The calculation parameters.

\begin{tabular}{|l|l|l|}
\hline Temperature of the electrolyte & ${ }^{\circ} \mathrm{C}$ & 956 \\
\hline Temperature of the environment & ${ }^{\circ} \mathrm{C}$ & 20 \\
\hline Temperature of the electrolyte liquidus & ${ }^{\circ} \mathrm{C}$ & 820 \\
\hline $\begin{array}{l}\text { Heat transfer coefficient: } \\
\text { melt / unit } \\
\text { cover / air }\end{array}$ & $\begin{array}{l}\mathrm{W} /\left(\mathrm{m}^{2} \cdot{ }^{\circ} \mathrm{C}\right) \\
\mathrm{W} /\left(\mathrm{m}^{2} \cdot{ }^{\circ} \mathrm{C}\right)\end{array}$ & $\begin{array}{l}830 \\
25\end{array}$ \\
\hline The density of the electrolyte & $\mathrm{kg} / \mathrm{m}^{3}$ & 2100 \\
\hline The viscosity of the electrolyte & $\mathrm{Pa} \cdot \mathrm{s}$ & $2,79 \cdot 10^{-3}$ \\
\hline The permeability of the bottom blocks & $\mathrm{m}^{2}$ & $10^{-13}$ \\
\hline
\end{tabular}

This problem is considered a two-phase filtration of incompressible immiscible liquids in an inhomogeneous non-deformable porous body. Assumed that the dynamic viscosities of the phases are constant, liquid flow is slow and occurs without phase transitions.

The computational domain is a half portion of electrolyzer cathode device, the geometry is shown on figure 2 . The weakest spot in electrolyzer construction are printed interblocks and peripheral seams, so the modeling was performed taking into account these features. In calculation takes into account all component parts of construction this aluminum electrolyzer, including interblock seams. Electrolyte filtration occurs through the porous structure of the bottom block and interblock seams. Mathematical modelling of electrolyte filtration was performed using CFD-pack FLUENT ver.14.

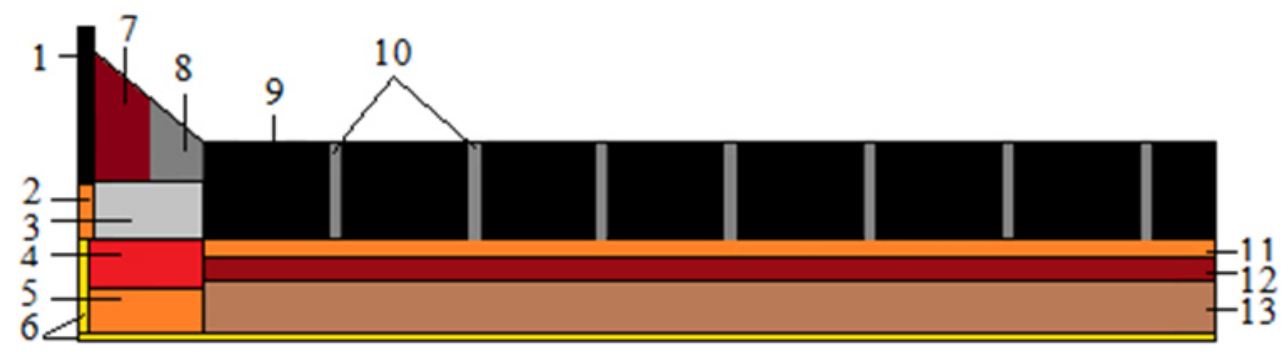

Figure 2. The geometry of the computational domain: 1 - SiC block, 2 - compensator, 3 - refractory concrete, 4 - refractory, 5 - heat insulation, 6 - leveling backfilling, 7 - inserter, 8 - peripheral seam, 9 - bottom block, 10 - interblock seams, 11 and 12 - refractory, 13 - heat insulation.

\section{Mathematical model}

Mathematical model of non-stationary filtration process and porous body heat transfer with incompressible viscous liquid consist of following equation system.

- liquid-flow equation in porous medium

$$
\vec{w}=-[D] \operatorname{grad} P
$$

- liquid continuity equation

$$
\frac{\partial \varepsilon \rho}{\partial \tau}+\operatorname{div}(\rho \vec{w})=0
$$

- energy equation for liquid

$$
(\rho c)_{\mathrm{G}} \varepsilon \frac{\partial T_{\mathrm{G}}}{\partial \tau}+\operatorname{div}\left((\rho \mathrm{c})_{\mathrm{G}} \vec{w} T_{\mathrm{G}}-\lambda_{\mathrm{G}} \operatorname{grad} T_{\mathrm{G}}\right)=\alpha_{\mathrm{V}}\left(T_{\mathrm{S}}-T_{\mathrm{G}}\right)
$$

Where the first term of equation on the left describes the increase of enthalpy, second term of equation heat convection, and on the right described heat transfer between liquid and solid body:

- heat-transfer equation in the porous medium 


$$
(\rho c)_{\mathrm{S}}(1-\varepsilon) \frac{\partial T_{\mathrm{S}}}{\partial \tau}+\operatorname{div}\left(-[\lambda]_{\mathrm{S}} \operatorname{grad}_{\mathrm{S}}\right)=\alpha_{\mathrm{V}}\left(T_{\mathrm{G}}-T_{\mathrm{S}}\right)+g_{\mathrm{V}}
$$

where:

$$
[\lambda]_{\mathrm{G}}=\left[\begin{array}{ccc}
\lambda_{1} & 0 & 0 \\
0 & \lambda_{2} & 0 \\
0 & 0 & \lambda_{3}
\end{array}\right]
$$

- effective heat-conduction tensor, recorder in the coordinate system $(\mathrm{X}, \mathrm{Y}, \mathrm{Z}) \mathrm{c}$ coincides with the main axes of the heat conduction, and $g_{\mathrm{v}}$ - volumetric heat generation in porous material;

- equation of condition for liquid

$$
\rho_{\mathrm{G}}=\rho\left(\rho, T_{\mathrm{G}}, \tau\right)
$$

In this article, to determine free boundary of the electrolyte used fluid-in-cell method, suggested by Hirt and Niholson [7]. This method is rightfully considered one of the most effective methods for solving problems with the free liquid surface [8-9]. The idea of this method is that the liquid and gas are considered as a unified two-component medium and the spatial distribution of phases within the limits the computational domain is defined by a special marker function $F(x, y, z, t)$, the value of which determines the volume ratio of liquid phase in computational cell as follows:

$$
f(x)=\left\{\begin{array}{l}
0, \\
1,
\end{array}\right\} \text { if the cell is empty }
$$

and $0<F(x, y, z, t)<1-$ if the phase boundary passes through cell.

Since the free surface of the electrolyte moves with liquid, then tracking free boundary moving in space accomplished by solving transport equation of liquid phase volume ratio in cell.

$$
\frac{\partial \varepsilon F}{\partial \tau}+\vec{w} \cdot \overrightarrow{\mathrm{V}} \varepsilon F=0
$$

Here $\vec{w}$ - velocity vector of two-phase medium, found by solving the system of equations of hydrodynamics. The density and molecular viscosity two-component medium are considered by the volume fraction of the liquid in the cell mixture according to the rule:

$$
\begin{aligned}
& \rho=\rho_{1} F+(1-F) \rho_{2}, \\
& \mu=\mu_{1} F+(1-F) \mu_{2} .
\end{aligned}
$$

Here $\rho_{1}, \mu_{1}-$ density and viscosity of electrolyte, $\rho_{2}, \mu_{2}$ - respectively, density and viscosity of air. Thus obtained density values $\rho$ and viscosity $\mu$ are included in the equation of motion and determine the physical properties of the two-phase medium.

\section{The verification of model}

To verify the developed model was solved the test, consisting in displacing oil by water from the composite porous body, and performed comparison calculations with experimental results [10]. The experimental setup is a tank with transparent walls. Inside the container was sprinkled glass beads, the space between them filled with oil. Upper and lower limit vessel size 20x10x0,6 cm impermeable, and the left and right sides of the flow. The flow domain is divided into four zones, characterized by absolute permeability coefficients $k_{1}=0.27 \mu \mathrm{m}^{2}$ and $k_{2}=0.11 \mu \mathrm{m}^{2}$. Porosity $\mathrm{m}=0.4$. Oil viscosity $\mu_{0}=5 \mathrm{mPa} \cdot \mathrm{s}$, the viscosity of water $\mu_{\mathrm{w}}=1$ $\mathrm{mPa} \cdot \mathrm{s}$. Through the left section in the resulting injection water reservoir at a rate of $\mathrm{Q}=1 \mathrm{ml} / \mathrm{min}$, and through the right section model selects liquid. The experimental setup is shown in figure 1 . The calculations were performed on a grid $100 \times 50$. The time step is equal to 0.1 . 


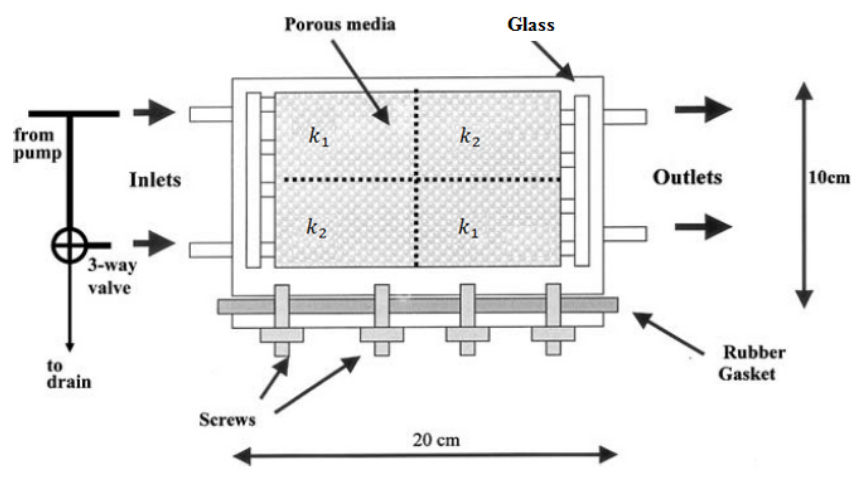

Figure 3. The experimental setup.

The experimental and calculated the current lines for the task are shown in Figure 4. As you can see the calculated current lines reproduce well the trajectory of the paint particles in the experiment. Fig. 5 shows the dynamics of the water filtration at time $t=2$, 5,8 and $11 \mathrm{~min}$. The results were visualized using red dye.

a.

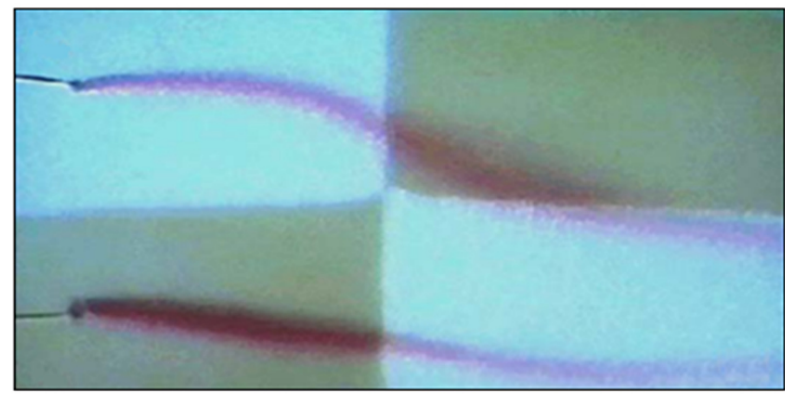

b.

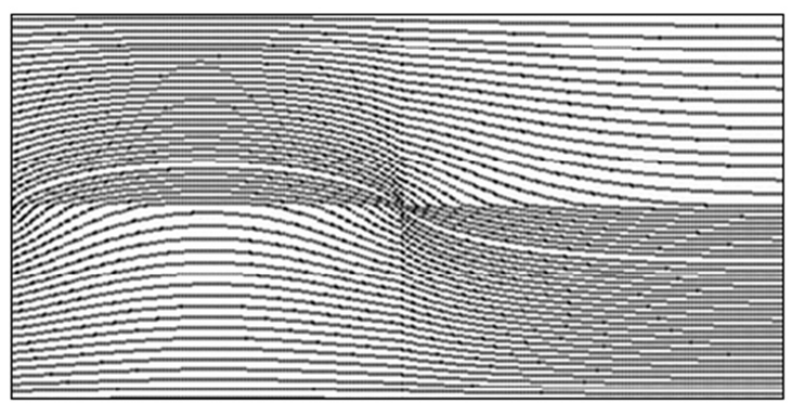

Figure 4. The stream lines. a - experiment, $b$ - calculation.

a.
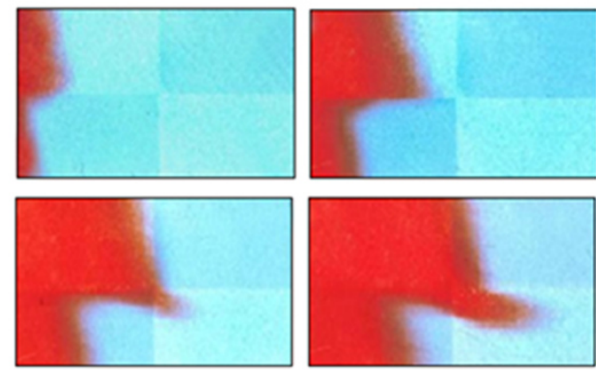

b.
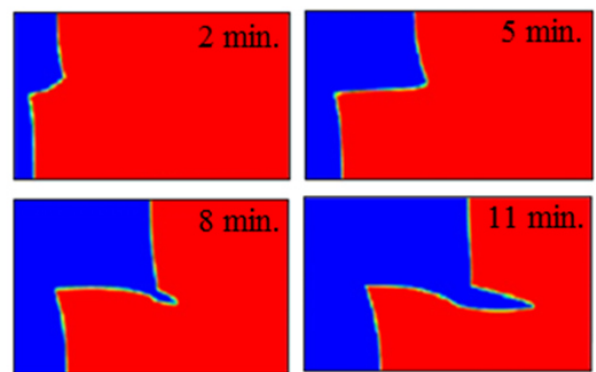

Figure 5. The water impregnation. a - experiment, b - calculation. 
Results show that the shape of the water free surface and it's dynamics in time, closely corresponded between calculation and experiment.

\section{The calculation results and discussion}

On figure 6 shown calculated temperature pattern in the bottom at different time from the beginning of filtration process.

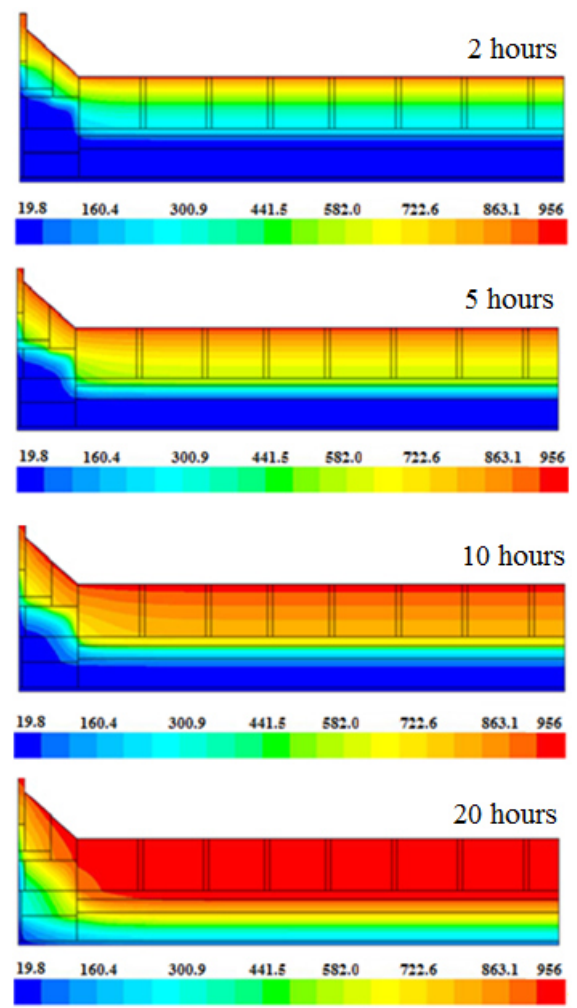

Figure 6. The temperature field in the cathode bottom.

Figure 6 shows that during the 20 hours since the start of operation of the cell electrolyte liquidus isotherm $\left(820^{\circ} \mathrm{C}\right)$ reaches a refractory layer and practically comes to insulation. Therefore, when the high permeability of bottom blocks melt eventually held at the same distance. The following calculation results impregnating the cathode (figure 8). Confirm this. After about 20-30 hours of the onset of the temperature field in the cathode becomes substantially stationary. By profile cathode observed temperature gradient in both the vertical and horizontal direction. It is evident that much warmed peripheral seal area, but this is due to the fact that at this stage in the formulation of the problem was not considered a protective layer of frozen electrolyte.

The following are results of calculations impregnation bottom blocks electrolyzer at different times. 

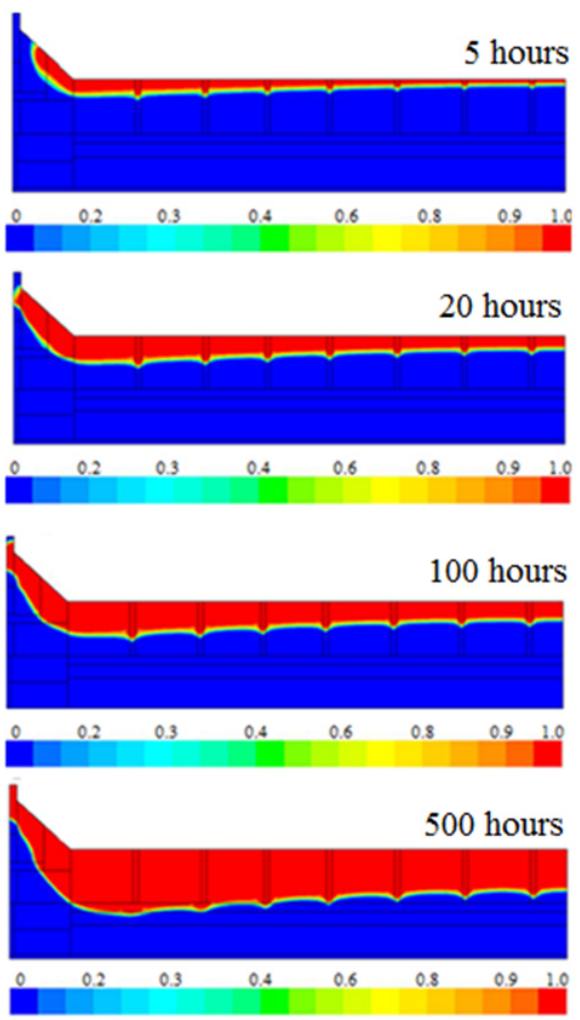

Figure 7. The cathode impregnation by electrolyte.

Figure 7 shows that the most heavily impregnated with electrolyte is in the first hours of operation, when the pressure gradient is the highest. During this time, there is a saturation of the upper layer block. Further, it is clear that the filtering of the electrolyte slows down, and the front impregnating gradually approaching the liquidus isotherm electrolyte. It is also evident that the impregnation extends in the vehicle unit. This is because in solving the heat problem was not considered a protective layer of the frozen electrolyte, which would prevent a rise in temperature in this area, thus protecting it from the penetration of the electrolyte. As the cathode is impregnated with electrolyte material increases the thermal conductivity and thus the liquidus temperature of the melt moves down which allows the electrolyte to penetrate further into the bath.

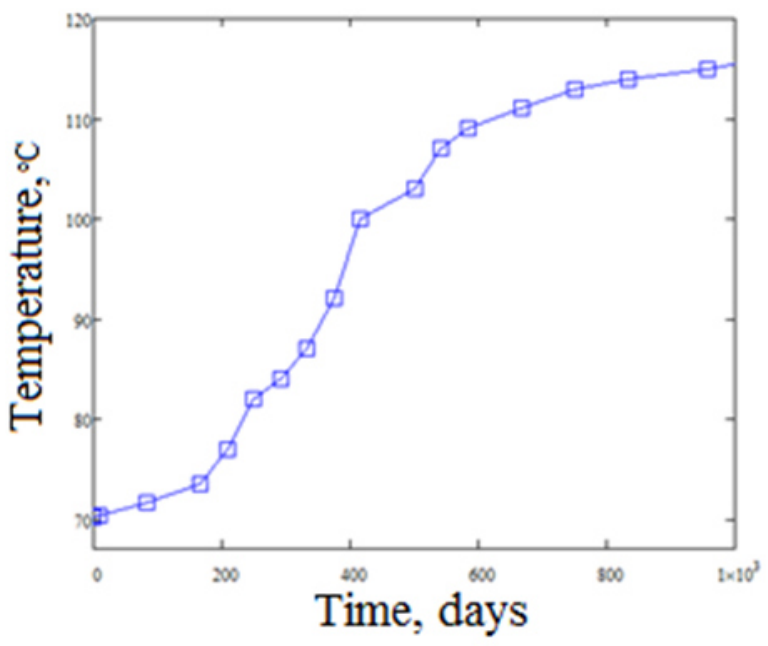

Figure 8. Changing the temperature on the bottom of the cathode unit cell.

Figure 8 is a graph of temperature rise at the bottom of the cathode unit cell. It can be seen that as the impregnation of the cathode increases temperature at the bottom of the cathode unit. It is planned to measure the temperature of the cathode unit in the active cell for the purpose of verification and adaptation of the developed mathematical model.

\section{Conclusions}

A mathematical model of heat transfer processes in the cathode device and filtering of the electrolyte in the bottom of the cathode. The verification model that confirms its adequacy. With the help of the developed mathematical model the dynamics of the impregnation of the lining of the cathode and electrolyte device defined thermal balance baths. Studies have shown how fast and how is the penetration of the melt in the bottom of the bath during operation of the cell. In the future, the results will help to 
better understand the processes of thermal penetration of the electrolyte in the cathode of the cell lining materials, and will provide an opportunity to optimize the structure of the lining to reduce heat costs and extend the life of the cathode of the device.

\section{Acknowledgment}

The investigation is supported by the Ministry of Education and Science of the Russian Federation Government contract with Siberian Federal University (No.3100).

\section{References}

1. Sorlie M., Oye H.A. Cathodes in Aluminium Electrolysis. 2nd edition. Aluminium-Vertag. 1994. 408 p.

2. Proshkin A. V., Pogodaev A.M., Polyakov P.V. et al. Property Change of Dry Barrier Mixes Used in Cathode of Aluminium Reduction Cell // Light Metals. 2007. P.833-838

3. M. V. Pervukhin, A. V. Minakov, N. V. Sergeev, M. U. Khatsauk. Mathematic simulation of electromagnetic and thermal hydrodynamic processes in the "inductor-ingot" system of an electromagnetic mould. Magnetohydrodynamics, vol. 47 (2011), No. 1, pp. 79-87.

4. Minakov, A. , Khatsayuk, M., Demidovich, V., Pervukhin, M. Mathematical modeling of casting processes in electromagnetic field. Magnetohydrodynamics. Volume 51, Issue 1, 2015, Pages 57-66

5. Shamsili R. and 0ye H. A. Melt Penetration and Chemical Reactions in Carbon Cathodes During Aluminium Electrolysis. 1П. // Light Metals. 1994. P.731-739.

6. Wang, Z., J. Rutlin, and T. Grande, Sodium diffusion in cathode lining in aluminium electrolysis cells. Light Metals (TMS), 2010: p. 841-847.

7. Hirt C.W. Volume of fluid (VOF). Method for the dynamics of free boundaries. / C.W. Hirt, B.D. Nichols // Journal of computational physics. 1981. № 39. P.-201-226.

8. A.V. Minakov. Numerical algorithm for moving-boundary fluid dynamics problems and its testing. Computational Mathematics and Mathematical Physics. Volume 54, Issue 10, 2014, P.- 1560-1570.

9. D. V. Guzei, A. V. Minakov, M. I. Pryazhnikov, A. A. Dekterev. Numerical modeling of gas-liquid flows in mini- and microchannels. Thermophysics and Aeromechanics January 2015, Volume 22, Issue 1, pp 61-71

10. Richard A. Dawe, Carlos A. Grattoni. Experimental displacement patterns in a $2 \times 2$ quadrant block with permeability and wettability heterogeneities_-problems for numerical modeling. Transp Porous Med. (2008) 71:5-22 